AperTO - Archivio Istituzionale Open Access dell'Università di Torino

\title{
Costly expertise
}

\section{This is the author's manuscript}

Original Citation:

Availability:

This version is available http://hdl.handle.net/2318/1723678

since 2020-01-16T19:11:27Z

Published version:

DOI:10.1257/aer.98.2.187

Terms of use:

Open Access

Anyone can freely access the full text of works made available as "Open Access". Works made available under a Creative Commons license can be used according to the terms and conditions of said license. Use of all other works requires consent of the right holder (author or publisher) if not exempted from copyright protection by the applicable law. 


\section{Costly Expertise \\ DinO GERARDi*AND LEEAT YARIV ${ }^{\dagger \ddagger}$}

December, 2007

In many environments expertise is costly. Costs can manifest themselves in numerous ways, ranging from the time that is required for a financial consultant to study companies' performances, to the resources necessary for academic referees to produce knowledgeable reports, to the attention and thought needed for jurors to construct informed convictions. The current paper asks a natural question germane to such contexts: how should a committee of potential experts be designed, in terms of the number of participants, their a-priori preferences, as well as the rules by which their recommendations are aggregated into a collective policy?

We consider a model in which a principal makes a binary decision (e.g., continue or abort a project), the value of which depends on the realization of some underlying state that is unknown (say, whether the project is great or inferior). The principal can hire a committee of experts from a pool varying in their preferences. All experts have access to an information technology providing (public) information regarding the underlying state. Information comes at a private cost to the experts, who care both about the final decision the principal takes, as well as about the amount they had personally spent on information acquisition.

Concentrating on small committees comprised of up to two potential experts, we provide several layers of responses to our fundamental design question, varying in the flexibility of the available contracts. First, we study institutions in which agents make their decisions regarding information acquisition simultaneously and characterize the optimal way to organize committees consisting of either one or two agents.

\footnotetext{
${ }^{*}$ Department of Economics, Yale University, e-mail: donato.gerardi@yale.edu

${ }^{\dagger}$ Division of Humanities and Social Sciences, California Institute of Technology, e-mail: lyariv@hss.caltech.edu

${ }^{\ddagger}$ Preliminary results on which this paper is based have been presented under the title "Organizational Design with Costly Information." We are grateful to audiences at the SISL conference, 2005 and the ASSA meetings, 2006. We also thank David Austen-Smith for a useful discussion of the paper.
} 
Next, we consider the full array of sequential mechanisms. We characterize the optimal sequential mechanism and identify the type of contract as well as the preferences of experts that are optimal across all classes of institutions (simultaneous or sequential). Namely, for a large class of cost functions, principals are divided into two types. For sufficiently moderate principals, a sequential mechanism with two identical experts who have opposing prior inclinations to the principal, and are more extreme, is optimal. For all other principals, none of the mechanisms are incentive compatible, and such principals are best off using no experts and following their ex-ante inclination.

Underlying the solution to our design problem (and any of its forms) are two trade-offs. First, for any expert preferences, there is a trade-off between the need to induce participants to acquire expertise on the one hand, and the desire to fully utilize this expertise on the other hand. Second, when choosing experts' preferences, there is a trade-off between choosing agents with similar preferences to the principal's in order to make revealing accurate information more valuable to the experts. On the other hand, choosing experts with more extreme preferences makes mistakes more costly for them and therefore induces more information acquisition as well.

In resolving the first trade-off, the optimal mechanism entails the use of at most two agents in a sequential mechanism, one in which two signals are collected only some of the time. In resolving the second trade-off, the optimal mechanism consists of agents who have opposing and more extreme preferences than the principal.

The analysis is useful in its applicability to institutional design in a wide range of environments, practically any in which information (that is later to be aggregated) is not fully exogenous. It is also important in eliciting the crucial differences between the optimal organizational designs that arise under these different plausible institutional structures.

The paper contributes to the recently growing literature on collective choice with endogenous information, including work by Calvert [1985], Li [2001], Cai [2003], Persico [2004], Dur and Swank [2005], Smorodinsky and Tennenholtz [2005], Gerardi and Yariv [2007a,b,c], Che and Kartik [2007], and Gershkov and Szentes [2008]. Our innovation is in the provision of a simple model allowing for the characterization of the optimal general mechanism entailing the committee's size and preference composition, as well as the rule by which the principal aggregates information (static or dynamic). 


\section{The MODEL}

There is a principal and a large population of experts who apply for two positions. All experts have the same ability but different preferences. The goal of the principal is to select the optimal mechanism and the optimal pair of experts. Our setup is reminiscent of the standard jury model (see, e.g., Austen-Smith and Banks, 1996 or Feddersen and Pesendorfer, 1998).

We focus on the case in which the two states, $I$ and $G$ (a metaphor for a project that is either inferior or great), are equally likely: $r \equiv \operatorname{Pr}(G)=1 / 2$.

Information is costly. An expert $j$ can acquire a signal of quality $p_{j} \in[1 / 2,1]$. The signal is binary, $s_{j}=i, g$. The probability distribution of a signal $s_{j}$ of quality $p_{j}$ is $\operatorname{Pr}\left(s_{j}=i \mid I\right)=\operatorname{Pr}\left(s_{j}=g \mid G\right)=p_{j}$. If more than one expert purchases information, we assume their signals are conditionally independent.

Let $j=1,2$ and fix a signal of quality $p_{j}$. Suppose that the realization of the signal is $s_{j}=$ $i, g$. We let $\operatorname{Pr}\left(\omega \mid s_{j} ; p_{j}\right)$ denote the probability that the state is $\omega=I, G$ given $p_{j}$ and $s_{j}$. Then, $\operatorname{Pr}\left(I \mid i ; p_{j}\right)=\operatorname{Pr}\left(G \mid g ; p_{j}\right)=p_{j}$. We also let $\operatorname{Pr}\left(\omega \mid s_{1}, s_{2} ; p_{1}, p_{2}\right)$ denote the probability that the state is $\omega=I, G$ given $p_{1}, p_{2}, s_{1}$ and $s_{2}$.

The cost of acquiring a signal of quality $p$ is $c(p)$. The cost function $c:[1 / 2,1] \rightarrow \mathbb{R}_{+}$satisfies the following properties:

$\cdot c^{\prime}\left(\frac{1}{2}^{+}\right)=0\left(c^{\prime}\left(\frac{1}{2}^{+}\right)\right.$is the right derivative of $c$ at $\left.p=1 / 2\right)$

. $c^{\prime}\left(1^{-}\right) \geqslant 1\left(c^{\prime}\left(1^{-}\right)\right.$is the left derivative of $c$ at $\left.p=1\right)$;

. $c^{\prime \prime}(p)>0$ and $c^{\prime \prime \prime}(p) \geqslant 0$ for every $p \in[1 / 2,1]$ (i.e., the marginal cost $c^{\prime}$ is increasing and convex).

We introduce an additional condition on $c$.

Condition 1. There exist two numbers, $\alpha$ and $\beta$ in $[0,1]^{2}$, such that $c^{\prime}\left(\frac{\alpha+\beta}{2}\right)>1 / 2$ and

$$
c^{\prime}(\alpha)=\beta, c^{\prime}(\beta)=\frac{\alpha}{2} .
$$

Notice that under our assumptions on the function $c$, the system (1) always admits a unique solution $(\alpha, \beta) \in[0,1]^{2}$. Thus, condition (1) imposes a restriction on the value of the cost function $c$ at the midpoint of the solutions to the system (1). 
Condition (1) is satisfied if the marginal cost $c^{\prime}$ has "enough curvature." For example, suppose that

$$
c^{\prime}(p)=\frac{p-1 / 2}{k(1-p)}
$$

where $k>0$. Then condition (1) is satisfied for $k$ sufficiently large.

The signals are public but the effort of the experts is not observable.

There are two decisions the principal can take: $A$ or $C$ (a metaphor for aborting or continuing the project). The principal's threshold of reasonable doubt is $q^{P}$ and for each $q \in[0,1]$ there are at least two experts with threshold $q$ (i.e., the principal can hire any pair of experts). We normalize the utility of the optimal decision to zero and set $u(A, G ; q)=-(1-q)$ and $u(C, I ; q)=-q$.

The case $q^{p}=r=1 / 2$ is special in the sense that adding a signal of quality $p$ to an existing signal of the same quality $p$ does not increase the principal's expected payoff. Thus we assume that $q^{P} \neq r$. In particular, we focus on the case $q^{P}>r=1 / 2$ (the principal cares more about the mistake of continuing an inferior project). Clearly, the optimal uninformed decision is $A$.

We assume that the principal does not have the ability to commit. In particular, given the available information, the principal chooses the action that maximizes her expected payoff. Because of this, we can restrict attention to the following mechanisms.

Mechanism with One Expert The principal hires one expert. The principal follows the expert's signal. That is, the principal chooses $A$ when the signal is $i$ and $C$ when the signal is $g$.

Simultaneous Mechanism The two experts acquire their signals simultaneously (i.e., an expert does not observe the realization of the signal of the other expert).

Sequential Mechanisms The relevant sequential mechanisms to consider are the following:

- Class $S_{i A}$ If the signal of the first expert is $i$ the principal chooses $A$ without consulting the second expert. If the first signal is $g$ the principal continues and follows the advice of expert 2 .

- Class $S_{g C}$ If the signal of the first expert is $g$ the principal chooses $C$ without consulting the second expert. If the first signal is $i$ the principal continues and follows the advice of expert 2 . 
- Class $S_{g A}$ If the signal of the first expert is $g$ the principal chooses $A$ without consulting the second expert. If the first signal is $i$ the principal continues and follows the advice of expert 2 .

- Class $S_{2}$ The principal always asks the second expert to invest and follows his signal.

Of course, the mechanism has to be incentive compatible. That is, given the available information (and the equilibrium strategies of the experts) it is optimal for the principal to follow the action prescribed by the mechanism. ${ }^{1}$ Our goal is to find the optimal mechanism and the optimal pair of experts. We evaluate the mechanism from the point of view of the principal. In particular, we do not take into account the experts' cost of acquiring information.

In principal, we should also consider mechanisms in which the principal randomizes at the action stage. These mechanisms are incentive compatible only if the principal is indifferent (i.e., her belief that the state is $G$ is equal to $q^{P}$ ). It is tedious but simple to show that random mechanisms are not optimal. Intuitively, adding randomness to a mechanism decreases the probability that the experts' signals are pivotal. This, in turn, lowers the incentives of the experts to acquire information. ${ }^{2}$

Under our assumptions we are able to characterize the optimal mechanism.

Proposition (Optimal Generalized Mechanisms) Whenever the cost function c satisfies Condition (1), there exists a threshold level $\bar{q}^{P} \in\left(\frac{1}{2}, 1\right)$ such that:

(i) If $q^{P} \leqslant \bar{q}^{P}$, then mechanism of class $S_{i A}$ with experts $q_{1}=q_{2}=0$ is optimal;

(ii) If $q^{P}>\bar{q}^{P}$, then no mechanism is incentive compatible. The principal will make the optimal uninformed decision A.

\footnotetext{
${ }^{1}$ For the sake of brevity, we do not consider mechanisms that would violate incentive compatibility directly. For example, consider the following mechanism. If the signal of the first expert is $i$ the principal chooses $C$ without consulting the second expert. If the first signal is $g$ the principal continues and follows the advice of expert 2 . The mechanism is not incentive compatible because it is not optimal for the principal to choose $C$ after observing the signal $i$ (recall that $\left.q^{P}>r\right)$.

${ }^{2}$ In principle, there is another form of randomness. A principal could choose a mechanism in which she randomly decides whether to consult a second expert or not. However, such mechanisms are not credible. Recall that the principal has no commitment power. If the second expert acquires information, the principal has a strict incentive to hire him. Note that in the mechanisms of class $S_{i A}, S_{g C}$, and $S_{g A}$ the principal does not have an incentive to consult the second expert when she is not supposed to. Indeed, it is enough to assume the following Nash equilibrium off-path behavior: the second expert does not acquire information and the principal does not react to any information collected.
} 
We provide the proof of the Proposition in the remaining sections, illustrating the optimal mechanism within each class on the way. ${ }^{3}$

\section{ONE EXPERT}

Consider a mechanism with one expert who has a threshold equal to $q$. If the principal has very extreme preferences, no individual expert can sway her prior inclinations, and she is best off choosing $A$ regardless of the expert's reports. In any other case, any expert would be useful, as the following claim illustrates.

Claim 1 (Optimal Mechanism with One Expert) If $q^{P} \leqslant\left(c^{\prime}\right)^{-1}\left(\frac{1}{2}\right)$, then any expert is optimal and the accuracy acquired is $\left(c^{\prime}\right)^{-1}\left(\frac{1}{2}\right)$. If $q^{P}>\left(c^{\prime}\right)^{-1}\left(\frac{1}{2}\right)$, then it is optimal for the principal to hire no expert and choose the action A (or hire an expert and disregard their advice, which in equilibrium will be uninformative).

Proof of Claim 1. Suppose that the principal follows the expert's signal. The expert's expected payoff if he chooses a signal of quality $p$ is equal to

$$
-r(1-q)(1-p)-(1-r) q(1-p)-c(p)=-\frac{1}{2}(1-p)-c(p)
$$

Notice that the expert's payoff does not depend on his threshold (since $r=1 / 2$ ). Of course, the expert will choose the level of effort $p_{0}$ such that $c^{\prime}\left(p_{0}\right)=\frac{1}{2}$. The mechanism is incentive compatible if and only if $p_{0}=\operatorname{Pr}\left(G \mid g, p_{0}\right) \geqslant q^{P}$, and the result follows.

Note that in much the same way, it is easy to see that if $r>1 / 2(r<1 / 2)$ then it is optimal to hire an expert with $q=0(q=1)$.

\section{Simultaneous Mechanism}

When two agents are employed, inducing both to acquire information is again contingent on the principal not being too extreme in her initial inclinations. In that case, their ideal preferences are more extreme than the principal's, as the following claim illustrates.

\footnotetext{
${ }^{3} \mathrm{~A}$ detailed proof appears in a working paper version, Gerardi and Yariv [2007c].
} 
Claim 2 (Optimal Simultaneous Mechanism) There is an incentive compatible mechanism with two agents acquiring information if and only if $q^{P} \leqslant \operatorname{Pr}(G \mid g, g ; \tilde{p}, \tilde{p})$, where $\frac{1}{2} \tilde{p}=c^{\prime}(\tilde{p})$. In that case, the optimal committee consists of two experts with $q=0$, who acquire signal accuracy $\tilde{p}$.

Proof of Claim 2. Without loss of generality we assume that in equilibrium expert 1 exerts more effort than expert 2 , i.e., $p_{1} \geqslant p_{2}>1 / 2$ (referred to as the (EQ) condition). The principal must choose $A$ after $(i, i)$ and $(i, g)$. Also, she must choose $C$ after $(g, g)$. Otherwise, she would always choose $A$ and the experts would not invest. Finally, the principal must choose $A$ after $(g, i)$. If not, then she would simply follow the advice of expert 1 and $p_{2}>1 / 2$ would not be optimal for expert 2 .

So we look for equilibria in which $p_{1} \geqslant p_{2}>1 / 2$ and the principal chooses $C$ if and only if the signal profile is $(g, g)$.

If $p_{1}, p_{2}$ are equilibrium efforts they have to satisfy:

$$
\begin{aligned}
& p_{1}=\arg \max _{p_{1}^{\prime}}-\frac{1}{2}\left(1-q_{1}\right)\left[1-p_{1}^{\prime} p_{2}\right]-\frac{1}{2} q_{1}\left[\left(1-p_{1}^{\prime}\right)\left(1-p_{2}\right)\right]-c\left(p_{1}^{\prime}\right) \text { and } \\
& p_{2}=\arg \max _{p_{2}^{\prime}}-\frac{1}{2}\left(1-q_{2}\right)\left[1-p_{1} p_{2}^{\prime}\right]-\frac{1}{2} q_{2}\left[\left(1-p_{1}\right)\left(1-p_{2}^{\prime}\right)\right]-c\left(p_{2}^{\prime}\right) .
\end{aligned}
$$

Combining the corresponding FOC's with the principal's IC constraints $\operatorname{Pr}\left(G \mid g, i ; p_{1}, p_{2}\right) \leqslant q^{P}$ and $\operatorname{Pr}\left(G \mid g, g ; p_{1}, p_{2}\right) \geqslant q^{P}$, we get the principal's problem to be:

$$
\max _{q_{1}, q_{2}, p_{1}, p_{2}}-\frac{1}{2}\left(1-q^{P}\right)\left[1-p_{1} p_{2}\right]-\frac{1}{2} q^{P}\left[\left(1-p_{1}\right)\left(1-p_{2}\right)\right]
$$

subject to the following constraints

$$
\begin{array}{ll}
\text { (FOC) } & \frac{1}{2}\left(1-q_{1}\right) p_{2}+\frac{1}{2} q_{1}\left(1-p_{2}\right)=c^{\prime}\left(p_{1}\right) \text { and } \frac{1}{2}\left(1-q_{2}\right) p_{1}+\frac{1}{2} q_{2}\left(1-p_{1}\right)=c^{\prime}\left(p_{2}\right) \\
\text { (IC) } & \frac{p_{1}\left(1-p_{2}\right)}{p_{1}\left(1-p_{2}\right)+\left(1-p_{1}\right) p_{2}} \leqslant q^{P} \text { and } \frac{p_{1} p_{2}}{p_{1} p_{2}+\left(1-p_{1}\right)\left(1-p_{2}\right)} \geqslant q^{P} \\
\text { (EQ) } & p_{1} \geqslant p_{2}>1 / 2
\end{array}
$$

Let $j=1,2$ and $k=3-j$ denote the pair of experts. We define the function $F_{j}\left(q_{j}, p_{k}\right)$ as follows:

$$
F_{j}\left(q_{j}, p_{k}\right)=\left(c^{\prime}\right)^{-1}\left(\frac{1}{2}\left(1-q_{j}\right) p_{k}+\frac{1}{2} q_{j}\left(1-p_{k}\right)\right)
$$


and notice that

$$
\frac{\partial F_{j}\left(q_{j}, p_{k}\right)}{\partial q_{j}}=\frac{\frac{1}{2}-p_{k}}{c^{\prime \prime}\left(F_{j}\left(q_{j}, p_{k}\right)\right)}<0 \text { and } \frac{\partial F_{j}\left(q_{j}, p_{k}\right)}{\partial p_{k}}=\frac{\frac{1}{2}-q_{j}}{c^{\prime \prime}\left(F_{j}\left(q_{j}, p_{k}\right)\right)}
$$

Suppose that both experts have $q=0$. The function $F_{j}\left(0, p_{k}\right)$ is increasing, $c^{\prime}\left(F_{j}\left(0, \frac{1}{2}\right)\right)=\frac{r}{2}=\frac{1}{4}$, and $c^{\prime}\left(F_{j}(0,1)\right)=r=\frac{1}{2}$. This implies that the functions $F_{1}(0, \cdot)$ and $F_{2}(0, \cdot)$ intersect (once because $F_{j}(0, \cdot)$ is concave). Moreover, if $\left(p_{1}, p_{2}\right) \neq\left(F_{1}\left(0, p_{2}\right), F_{2}\left(0, p_{1}\right)\right)$ is such that $p_{1} \leqslant F_{1}\left(0, p_{2}\right)$ and $p_{2} \leqslant F_{2}\left(0, p_{1}\right)$, then there exists a pair $\left(p_{1}^{\prime}, p_{2}^{\prime}\right)$ such that for every $j=1,2, p_{j}^{\prime}>p_{j}$ and $p_{j}^{\prime}=F_{j}\left(0, p_{k}^{\prime}\right)$.

Consider a pair $\left(q_{1}, q_{2}\right) \neq(0,0)$ and the corresponding equilibrium efforts $\left(p_{1}, p_{2}\right)$ with $p_{j}=$ $\left(F_{j}\left(q_{j}, p_{k}\right)\right)$. Notice that $F_{j}\left(q_{j}, p_{k}\right)<F_{j}\left(0, p_{k}\right)$ if $q_{j}>0$ since $F_{j}$ is decreasing in $q_{j}$. But then $\left(p_{1}, p_{2}\right) \neq\left(F_{1}\left(0, p_{2}\right), F_{2}\left(0, p_{1}\right)\right)$ and $p_{1} \leqslant F_{1}\left(0, p_{2}\right), p_{2} \leqslant F_{2}\left(0, p_{1}\right)$. We conclude that there exists $\left(p_{1}^{\prime}, p_{2}^{\prime}\right)=\left(F_{1}\left(0, p_{2}^{\prime}\right), F_{2}\left(0, p_{1}^{\prime}\right)\right)$ with $p_{j}^{\prime}>p_{j}$ for every $j$. The principal's utility is increasing in $p_{1}$ and $p_{2}$ and, thus, the pair of experts $(0,0)$ is optimal.

Consider now the FOC's for two experts with $q=0: \frac{1}{2} p_{2}=c^{\prime}\left(p_{1}\right)$ and $\frac{1}{2} p_{1}=c^{\prime}\left(p_{2}\right)$, which implies $\frac{1}{2} p_{2}+c^{\prime}\left(p_{2}\right)=\frac{1}{2} p_{1}+c^{\prime}\left(p_{1}\right)$. Obviously, the solution must be symmetric: $p_{1}=p_{2}=\tilde{p}$ satisfying $\frac{1}{2} \tilde{p}=c^{\prime}(\tilde{p})$. Since the effort is symmetric, $\operatorname{Pr}(G \mid g, i ; \tilde{p}, \tilde{p})=\frac{1}{2}<q^{P}$ and the claim follows.

In the next section we show that if there exists an incentive compatible simultaneous mechanism then there is also an incentive compatible sequential mechanism of class $S_{i A}$ which yields the principal a higher utility.

\section{Sequential Mechanisms}

We start with a heuristic comparison between the different sequential mechanisms. First, it is easy to check that in a mechanism of class $S_{g A}$ expert 1 does not acquire information. This is intuitive since the principal will either go against expert 1's signal, or ignore his advice altogether.

Furthermore, note that from the structure of the problem, $S_{i A}$ can be implemented with experts of preferences $\left(q_{1}, q_{2}\right)$ investing in information of accuracies $\left(p_{1}, p_{2}\right)$ if and only if $S_{g C}$ can be implemented with the same accuracies $\left(p_{1}, p_{2}\right)$ when choosing experts with preferences $\left(1-q_{1}, 1-q_{2}\right)$. 
Given that the principal is inclined toward a choice of $A$, upon choosing a sequential mechanism, it is sensible for her to pursue more information when preliminary evidence goes against her prior inclinations. That is, $S_{i A}$ generates greater expected payoffs than $S_{g C}$.

Comparing between the mechanism $S_{2}$ and $S_{i A}$ requires more subtle arguments. Indeed, when $S_{2}$ is implemented, the second expert in line conditions his level of investment on the signal reported by the first expert (otherwise, the first expert does not invest in information and we are back to the single expert case). Suppose that the second expert invests differential amounts depending on the first expert's signal realization. The accuracy acquired by the second agent is a random variable, with a distribution determined by the investment of the first expert. For sufficiently convex cost functions, the average accuracy is far lower than in $S_{i A}$, and generates a lower expected payoff. In fact, Condition (1) assures that this is indeed the case.

The above arguments can be formalized in a straightforward manner and their details appear in Gerardi and Yariv [2007c]. In what follows, we characterize the optimal committee when $S_{i A}$ is implemented and illustrate its superiority over the single agent as well as the simultaneous mechanism, thereby providing a proof for the Proposition.

Consider then the sequential mechanism $S_{i A}$ and let $p_{1}$ and $p_{2}$ denote the effort of the two experts. If expert 1 observes signal $g$, then expert 2 assigns probability $p_{1}=\operatorname{Pr}\left(G \mid g ; p_{1}\right)$ to state $G$.

The principal's utility is equal to

$$
-\frac{1}{2}\left(1-q^{P}\right)\left(1-p_{1} p_{2}\right)-\frac{1}{2} q^{P}\left(1-p_{1}\right)\left(1-p_{2}\right)
$$

and is increasing in $p_{1}$ and $p_{2}$.

The utility of expert 1 is equal to $-\frac{1}{2}\left(1-q_{1}\right)\left(1-p_{1} p_{2}\right)-\frac{1}{2} q_{1}\left(1-p_{1}\right)\left(1-p_{2}\right)-c\left(p_{1}\right)$ with FOC $\frac{1}{2}\left(1-q_{1}\right) p_{2}+\frac{1}{2} q_{1}\left(1-p_{2}\right)-c^{\prime}\left(p_{1}\right)=0$.

Recall that the function $F_{1}\left(q_{1}, p_{2}\right)$ is defined as follows:

$$
F_{1}\left(q_{1}, p_{2}\right)=\left(c^{\prime}\right)^{-1}\left(\frac{1}{2}\left(1-q_{1}\right) p_{2}+\frac{1}{2} q_{1}\left(1-p_{2}\right)\right)
$$

The utility of expert 2 is equal to $-\left[p_{1}\left(1-q_{2}\right)+\left(1-p_{1}\right) q_{2}\right]\left(1-p_{2}\right)-c\left(p_{2}\right)$ with FOC 
$p_{1}\left(1-q_{2}\right)+\left(1-p_{1}\right) q_{2}-c^{\prime}\left(p_{2}\right)=0$.

Let the function $G_{2}\left(q_{2}, p_{1}\right)$ be defined by

$$
G_{2}\left(q_{2}, p_{1}\right)=\left(c^{\prime}\right)^{-1}\left(p_{1}\left(1-q_{2}\right)+\left(1-p_{1}\right) q_{2}\right)
$$

and notice that

$$
\frac{\partial G_{2}\left(q_{2}, p_{1}\right)}{\partial q_{2}}=\frac{1-2 p_{1}}{c^{\prime \prime}\left(G_{2}\left(q_{2}, p_{1}\right)\right)}<0 \text { and } \frac{\partial G_{2}\left(q_{2}, p_{1}\right)}{\partial p_{1}}=\frac{\left(1-2 q_{2}\right)}{c^{\prime \prime}\left(G_{2}\left(q_{2}, p_{1}\right)\right)}
$$

Consider the function $G_{2}\left(0, p_{1}\right)=\left(c^{\prime}\right)^{-1}\left(p_{1}\right)$. It is increasing, $c^{\prime}\left(G_{2}\left(0, \frac{1}{2}\right)\right)=r=\frac{1}{2}$, and $c^{\prime}\left(G_{2}(0,1)\right)=1$.

This together with the properties of $F_{1}\left(0, p_{2}\right)$ (see proof of Claim 2) imply the following. The functions $F_{1}(0, \cdot)$ and $G_{2}(0, \cdot)$ intersect once. Moreover, if $\left(p_{1}, p_{2}\right) \neq\left(F_{1}\left(0, p_{2}\right), G_{2}\left(0, p_{1}\right)\right)$ is such that $p_{1} \leqslant F_{1}\left(0, p_{2}\right)$ and $p_{2} \leqslant G_{2}\left(0, p_{1}\right)$, then there exists a pair $\left(p_{1}^{\prime}, p_{2}^{\prime}\right)=\left(F_{1}\left(0, p_{2}^{\prime}\right), G_{2}\left(0, p_{1}^{\prime}\right)\right)$ with $p_{j}^{\prime}>p_{j}$ for every $j=1,2$.

We now show that the optimal pair of experts in a mechanism of class 1 is $q_{1}=q_{2}=0$.

Consider the pair $\left(q_{1}, q_{2}\right) \neq(0,0)$ with effort levels $\left(p_{1}, p_{2}\right)$. Notice that

$$
p_{1}=F_{1}\left(q_{1}, p_{2}\right) \leqslant F_{1}\left(0, p_{2}\right) \text { and } p_{2}=G_{2}\left(q_{2}, p_{1}\right) \leqslant G_{2}\left(0, p_{1}\right)
$$

and at least one inequality is strict. Thus, there exist effort levels $\left(p_{1}^{\prime}, p_{2}^{\prime}\right)$ for the pair of experts $(0,0)$ with $p_{j}^{\prime}>p_{j}$, for every $j$.

Let $\left(\hat{p}_{1}, \hat{p}_{2}\right)$ be the effort levels of the optimal pair of experts. They are defined by:

$$
\frac{1}{2} \hat{p}_{2}=c^{\prime}\left(\hat{p}_{1}\right) \text { and } \hat{p}_{1}=c^{\prime}\left(\hat{p}_{2}\right)
$$

Notice that under our assumptions ( $c$ convex, $\left.c^{\prime}(1 / 2)=0, c^{\prime}(1) \geqslant 1\right)$ the system of equations $(2)$ always admits a unique solution $1 / 2<\hat{p}_{1}<\hat{p}_{2}<1$.

Of course, we need to check that $\operatorname{Pr}\left(G \mid g, g ; \hat{p}_{1}, \hat{p}_{2}\right)>q^{P}$, otherwise the principal will not choose $C$ after receiving reports $(g, g)$ (it is straightforward to see that all the other constraints are satisfied). 


\section{Comparison between Simultaneous Mechanisms and $S_{i A}$ Mechanisms}

Suppose that there exists an incentive compatible simultaneous mechanism (with $q_{1}=q_{2}=0$ ) with symmetric effort level $\tilde{p}$ defined in Claim 2. Then it is easy to see that there is also an incentive compatible sequential mechanism of type $S_{i A}$ (with $q_{1}=q_{2}=0$ ) with effort levels $\hat{p}_{1}>\tilde{p}$ and $\hat{p}_{2}>\tilde{p}$. Recall also that in the optimal simultaneous mechanism the principal chooses $C$ if and only if both experts observe $g$. We conclude that the optimal mechanism of type $S_{i A}$ dominates the best simultaneous mechanism with two experts. ${ }^{4}$

\section{Comparison between One Expert Mechanisms and $S_{i A}$ Mechanisms}

Suppose that there exists an incentive compatible mechanism with one expert with effort level $p_{0}$ (defined in Claim 1). Then there also exists an incentive compatible mechanism of type $S_{i A}$ (with $q_{1}=q_{2}=0$ ) with effort levels $\hat{p}_{1}$ and $\hat{p}_{2}$. It follows from the definition of $p_{0}$ and the FOC's corresponding to $S_{i A}$ that $\hat{p}_{2}>p_{0}>\hat{p}_{1}$.

Let $U\left(\hat{p}_{1}, \hat{p}_{2}\right)$ denote the principal's expected payoff under the mechanism of class $S_{i A}$. Similarly, let $U\left(p_{0}\right)$ denote the principal's payoff under the mechanism with one expert. We have

$$
\begin{gathered}
U\left(\hat{p}_{1}, \hat{p}_{2}\right)=-\frac{1}{2}\left(1-q^{P}\right)\left(1-\hat{p}_{1} \hat{p}_{2}\right)-\frac{1}{2} q^{P}\left(1-\hat{p}_{1}\right)\left(1-\hat{p}_{2}\right), \\
U\left(p_{0}\right)=-\frac{1}{2}\left(1-q^{P}\right)\left(1-p_{0}\right)-\frac{1}{2} q^{P}\left(1-p_{0}\right) .
\end{gathered}
$$

After algebraic manipulations, the payoff difference between the two mechanisms is equal to

$$
\begin{gathered}
U\left(\hat{p}_{1}, \hat{p}_{2}\right)-U\left(p_{0}\right)=-\frac{1}{2}\left(1-q^{P}\right)\left(p_{0}-\hat{p}_{1} \hat{p}_{2}\right)+\frac{1}{2} q^{P}\left(\hat{p}_{1}+\hat{p}_{2}-\hat{p}_{1} \hat{p}_{2}-p_{0}\right)> \\
>-\frac{1}{2}\left(1-q^{P}\right)\left(p_{0}-\hat{p}_{1} \hat{p}_{2}\right)+\frac{1}{2}\left(1-q^{P}\right)\left(\hat{p}_{1}+\hat{p}_{2}-\hat{p}_{1} \hat{p}_{2}-p_{0}\right)=\frac{1}{2}\left(1-q^{P}\right)\left(\hat{p}_{1}+\hat{p}_{2}-2 p_{0}\right),
\end{gathered}
$$

where the inequality follows from the fact that $\left(\hat{p}_{1}+\hat{p}_{2}-\hat{p}_{1} \hat{p}_{2}-p_{0}\right)>0$ and $q^{P}>1-q^{P}$.

Notice that $\left(\hat{p}_{1}, \hat{p}_{2}\right)$ are the solution to the system of equations (2). It follows from Condition (1) and the definition of $p_{0}$ that

$$
c^{\prime}\left(\frac{\hat{p}_{1}+\hat{p}_{2}}{2}\right)>\frac{1}{2}=c^{\prime}\left(p_{0}\right)
$$

Recall that $c^{\prime}$ is strictly increasing. Thus, $\frac{\hat{p}_{1}+\hat{p}_{2}}{2}>p_{0}$. In particular, $U\left(\hat{p}_{1}, \hat{p}_{2}\right)>U\left(p_{0}\right)$ as needed.

\footnotetext{
${ }^{4}$ Note that this comparison relies only on the assumption that the cost function $c$ is convex, and does not require Condition (1).
} 


\section{REFERENCES}

[1] Austen-Smith, D. and Banks, J. S. [1996], "Information Aggregation, Rationality, and the Condorcet Jury Theorem," American Political Science Review, Volume 90(1), pages 34-45.

[2] Cai, H. [2003], "Optimal Committee Design with Heterogeneous Preferences," mimeo.

[3] Calvert, R. L. [1985], "The Value of Biased Information: A Rational Choice Model of Political Advice," The Journal of Politics, Volume 47(2), pages 530-555.

[4] Che, Y-K. and Kartik, N. [2007], "Opinions as Incentives," mimeo.

[5] Dur, R. and Swank , O. H. [2005], "Producing and Manipulating Information," Economic Journal, Volume 115, pages 185-199.

[6] Feddersen, T. J. and Pesendorfer, W. [1998], "Convicting the Innocent: The Inferiority of Unanimous Jury Verdicts under Strategic Voting," American Political Science Review, Volume 92(1), pages 23-35.

[7] Gerardi, D. and Yariv, L. [2007a], "Information Acquisition in Committees," Games and Economic Behavior, forthcoming.

[8] Gerardi, D. and Yariv, L. [2007b], "Deliberative Voting," Journal of Economic Theory, Volume 134, pages 317-338.

[9] Gerardi, D. and Yariv, L. [2007c], "Costly Expertise," http://www.hss.caltech.edu/ ^lyariv.

[10] Gershkov, A. and Szentes, B. [2008], "Optimal Voting Scheme with Costly Information Acquisition," Journal of Economic Theory, forthcoming.

[11] Li, H. [2001], "Theory of Conservatism," Journal of Political Economy, Volume 109(3), pages 617-636.

[12] Persico, N. [2004], "Committee Design with Endogenous Information," The Review of Economic Studies, Volume 71(1), pages 165-94.

[13] Smorodinsky, R. and Tennenholtz, M. [2005], "Overcoming Free Riding in Multi-Party Computations The Anonymous Case," Games and Economic Behavior, forthcoming. 